\title{
Neurophysiologic pain response in patients with juvenlie idiopathic arthritis - a pilot study
}

\author{
Johanne Marie M Iversen ${ }^{1 *}$, Ellen Dalen Arnstad ${ }^{1,2}$, Trond Sand ${ }^{3,4}$, Marite Rygg ${ }^{1,5}$ \\ From 21st European Pediatric Rheumatology (PReS) Congress \\ Belgrade, Serbia. 17-21 September 2014
}

\section{Introduction}

Pain is a common symptom in children and adolescents with JIA. It has been hypothesized that frequent pain experiences sensitize pain processing pathways, resulting in hypersensitivity to later painful stimuli. A lower pain threshold (PT) and pain tolerance in JIA patients have been demonstrated in previous studies using pressure algometry and the cold pressor task.

\section{Objectives}

To investigate pain thresholds in adolescents with JIA compared to age and sex matched healthy controls, using several modalities for experimental pain testing.

\section{Methods}

Consecutive adolescents with JIA (16-18 years) were recruited from the pediatric rheumatology outpatient clinic at St. Olavs Hospital. Healthy controls were recruited from a local upper secondary school. Both completed a validated questionnaire on health and quality of life (SF-36), and reported pain from the last week (VAS scale). Quantitative sensory testing was conducted, and thermal detection pain thresholds (PTs) recorded. A thermal element was held against three specified locations of the participant's skin, and the participant was instructed to press a button when he/she felt changes in temperature or pain. Pressure algometry was performed on two welldefined anatomical areas, giving the pressure pain threshold (PPT).

\section{Results}

Compared to 19 healthy controls, the 14 patients with JIA reported more pain during the last week, and had a less favorable score in the physical SF-36 domains, but no difference in the mental health domains. They displayed a lower PPT, but similar cold and warmth PT compared to the controls. When subdividing JIA patients with active and inactive disease, patients with inactive disease had a lower cold PT and PPT, and a tendency towards a lower heat PT compared to controls. Patients with active disease had a tendency towards higher PTs in all three modalities compared to both healthy adolescents and patients with inactive disease.

\section{Conclusion}

Our results indicate that JIA patients may be subject to a sensitization, giving lower pain thresholds in inactive disease, but once the disease is active, painful arthritis may act as a diversion leading to increased rather than lowered PT.

\section{Disclosure of interest \\ None declared.}

\section{Authors' details}

'Department of Laboratory Medicine, Children's and Women's Health, Norwegian University of Science and Technology, Trondheim, Norway. ${ }^{2}$ Department of Pediatrics, Hospital of Levanger, Levanger, Norway. ${ }^{3}$ Department of Neuroscience, Norwegian University of Science and Technology, Trondheim, Norway. ${ }^{4}$ Department of Neurology and Clinical Neurophysiology, University Hospital of Trondheim, Trondheim, Norway. ${ }^{5}$ Department of Pediatrics, St. Olavs Hospital, University Hospital of Trondheim, Trondheim, Norway.

Published: 17 September 2014

doi:10.1186/1546-0096-12-S1-P147

Cite this article as: Iversen et al:: Neurophysiologic pain response in patients with juvenlie idiopathic arthritis - a pilot study. Pediatric Rheumatology 2014 12(Suppl 1):P147. 